\title{
Erratum to: Emerging technologies in coloproctology: results of the Italian Society of Colorectal Surgery Logbook of Adverse Events
}

L. Basso $\cdot$ M. Pescatori $~$ F. La Torre $~$

I. Destefano $\cdot$ A. Pulvirenti D'Urso •

A. Infantino $\cdot$ A. Amato $\cdot$ AECO Group

Published online: 20 December 2012

(C) Springer-Verlag Italia 2012

Erratum to: Tech Coloproctol

DOI 10.1007/s10151-012-0906-6

Unfortunately reference 7 of this article was rendered incorrectly. It should have read as follows:

7. Basso L, Giuliani A (2011) On overdiagnosis and overtreatment of rectal conditions. BMJ. http://www.bmj.com/ rapid-response/2011/11/03/overdiagnosis-and-overtreatmentrectal-conditions

The online version of the original article can be found under doi:10.1007/s10151-012-0906-6.

L. Basso · M. Pescatori · F. La Torre - I. Destefano . A. P. D'Urso - A. Infantino - A. Amato - AECO Group Italian Society of Colorectal Surgery or Società Italiana di Chirurgia Colorettale (SICCR), Rome, Italy

L. Basso $(\bowtie)$

Department of Surgery, Faculty of Medicine and Dentistry,

"Sapienza" University of Rome, "Pietro Valdoni", Policlinico

"Umberto I", viale del Policlinico 155, 00161 Rome, Italy

e-mail: luigi.basso@uniroma1.it 